\title{
Supertranslations to All Orders*
}

\author{
Rainer Dick \\ Department of Physics and Engineering Physics, University of Saskatchewan, Saskatoon, Canada \\ E-mail: rainer.dick@usask.ca \\ Received December 29, 2009; revised February 25, 2010; accepted March 18, 2010
}

\begin{abstract}
We calculate the transformation laws of the general linear superfield $V(x, \theta, \bar{\theta})$ and chiral superfields under $N=1$ supertranslations $\exp [i(\zeta \cdot Q+\bar{Q} \cdot \bar{\zeta})]$ to all orders in the translation parameters $\zeta, \bar{\zeta}$. We use the superfield formalism with complete expansions of the component fields in the coordinate shifts $\Delta x^{\mu}=-i\left(\zeta \cdot \sigma^{\mu} \cdot \bar{\theta}-\theta \cdot \sigma^{\mu} \cdot \bar{\zeta}\right)$. The results show in particular how a general supertranslation transforms each component field of a supermultiplet into a complete superfield. The results also provide complete parametrizations of orbits of component fields under supertranslations.
\end{abstract}

Keywords: Supersymmetry, Supermultiplets, Supertranslations

\section{Introduction}

Quantum field theories with exact correspondences between bosonic and fermionic helicity states are not only basic ingredients for superstring theories, but have dominated both theoretical investigations and experimental searches for particle physics beyond the current "Standard Model" of particle physics for over three decades now.

The minimal version of supersymmetric extensions of the Standard Model extends the generators $M_{\mu v}, p_{\mu}$ of the Poincaré group by a set of fermionic generators $Q_{\alpha}$ and $\bar{Q}_{\dot{\alpha}}$ in the $(1 / 2,0)$ and $(0,1 / 2)$ representations of the proper orthochronous Lorentz group in four dimensions. It has been recognized early on that this extension of the Poincaré algebra can be represented linearly (and in a reducible, but not fully reducible manner) on a set comprising 4 complex spin-0 fields, 4 Weyl spinors and one complex spin-1 field. This set constitutes the so called general linear multiplet or general linear superfield $V$ and its irreducible subsets had also been identified.

It is sufficient to know the action of the supertranslation generators $Q_{\alpha}$ and $\bar{Q}_{\dot{\alpha}}$ on the components of $V$, or equivalently the action of the supertranslation $\exp [i(\zeta \cdot Q+\bar{Q} \cdot \bar{\zeta})]$ to first order in the parameters $\zeta_{\alpha}$, $\bar{\zeta}_{\dot{\alpha}}$, to construct supersymmetric action principles and

*This work was supported by NSERC Canada. the related supercurrents. Therefore the first order transformation laws for the components of $V$ have been calculated a long time ago and can be found in many books and review articles on supersymmetry and with our current understanding this is all that is needed to discuss the physical implications of supersymmetry. Recent research in supersymmetry focuses on important applications like new solutions and structure of supergravity theories [1-5], impact of supersymmetry on perturbative calculations [6], cosmological implications of supersymmetry [7-9] and in particular the interesting problem how supersymmetry can be reconciled with a cosmological constant and help to explain it $[10,11]$. The structure of superpotentials in theories with broken supersymmetry is also an active area of research with phenomenological relevance [12-14].

From a mathematical point of view it is clearly desirable to also have the full transformation properties of the general linear multiplet readily available for reference. To provide such a reference is the purpose of this paper. To make these results also easily accessible for beginners in supersymmetry, the super-Poincaré algebra and the basic techniques of superspace calculations are also reviewed. Therefore the outline of the paper is as follows.

Our conventions for spinor representations of the Lorentz group and the super-Poincaré algebra are introduced in Section 2. Superspace is reviewed in Section 3 and the full supertranslation properties of the component fields of the general linear multiplet are calculated in Section 4 
Chiral superfields provide a particular irreducible representation within the reducible linear multiplet. Due to their practical relevance for the supersymmetrization of matter fields, the resulting supertranslation properties of the components of chiral superfields are listed in Section 5.

Appendix 1 contains a translation of our results into the conventions of Wess and Bagger [15]. The relevant spinor indices are reviewed in Appendix 2.

Our conventions for spinor representations and superspace calculations differ from Wess and Bagger only with regard to the definition of superderivatives and the definition of the $2^{\text {nd }}$ order epsilon spinors with lower indices. Sections 2 and 3 are included to make the paper self-contained and easily accessible and to clarify conventions. However, the new results in Sections 4 and 5 are not affected by the different definitions. The cognoscenti should therefore go straight to Section 4.

\section{The Super-Poincaré Algebra}

The basic methodology for calculations with linear super-multiplets in four dimensions was developed some 35 years ago by Wess, Zumino, Salam, Strathdee and Ferrara [16-19]. This section and the following section provide a brief but concise introduction to the calculational techniques of supersymmetry and its linear representations in four dimensions.

We use $\eta_{00}=-1$ for the Minkowski metric and standard notation $\sigma_{\mu \alpha \dot{\alpha}}$ with

$$
\begin{aligned}
& \sigma_{0}=\left(\sigma_{0 \alpha \dot{\alpha}}\right)=\left(\begin{array}{cc}
1 & 0 \\
0 & 1
\end{array}\right), \quad \sigma_{1}=\left(\sigma_{1 \alpha \dot{\alpha}}\right)=\left(\begin{array}{ll}
0 & 1 \\
1 & 0
\end{array}\right), \\
& \sigma_{2}=\left(\sigma_{2 \alpha \dot{\alpha}}\right)=\left(\begin{array}{cc}
0 & -i \\
i & 1
\end{array}\right), \quad \sigma_{3}=\left(\sigma_{3 \alpha \dot{\alpha}}\right)=\left(\begin{array}{cc}
1 & 0 \\
0 & -1
\end{array}\right)
\end{aligned}
$$

for the Pauli matrices.

Complex conjugation turns undotted indices into dotted indices and vice versa,

$$
\left(\psi_{a}\right)^{*}=\bar{\psi}_{\dot{\alpha}},
$$

and hermiticity of the Pauli matrices implies for the complex conjugate matrices

$$
\bar{\sigma}_{\dot{\alpha} \alpha}^{\mu}=\sigma_{\alpha \dot{\alpha}}^{\mu} .
$$

We pull spinor indices with the two-dimensional epsilon spinors

$$
\begin{array}{ll}
\varepsilon^{12}=\varepsilon_{12}=1, & \varepsilon^{i \dot{2}}=\varepsilon_{i 2}=1, \\
\psi^{\alpha}=\varepsilon^{\alpha \beta} \psi_{\beta}, & \psi_{\beta}=\psi^{\alpha} \varepsilon_{\alpha \beta}, \\
\bar{\psi}^{\dot{\alpha}}=\varepsilon^{\dot{\alpha} \dot{\beta}} \bar{\psi}_{\dot{\beta}}, & \bar{\psi}_{\dot{\beta}}=\bar{\psi}^{\dot{\alpha}} \varepsilon_{\dot{\alpha} \dot{\beta}} .
\end{array}
$$

The Equations (1) then imply that the conjugate Pauli matrices with upper spinor indices are

$$
\bar{\sigma}^{\mu \dot{\alpha} \alpha}=\varepsilon^{\dot{\alpha} \dot{\beta}} \varepsilon^{\alpha \beta} \sigma_{\beta \dot{\beta}}^{\mu} .
$$

Numerically, we have with the upper index positions for the barred matrices and lower index positions for the unbarred matrices

$$
\bar{\sigma}_{0}=\sigma_{0}, \quad \bar{\sigma}_{i}=-\sigma_{i} .
$$

Although not formally required, use of upper indices for barred Pauli matrices and lower indices for unbarredPauli matrices is a useful and very common convention.

Relations for Pauli matrices are meticulously compiled in [15]. For convenience, we recall those relations which are directly relevant for the derivation of supertranslations to all orders,

$$
\begin{gathered}
\bar{\sigma}_{\mu}{ }^{\dot{\alpha} \alpha} \sigma_{\beta \dot{\beta}}^{\mu}=-2 \delta_{\dot{\beta}}^{\dot{\alpha}} \delta_{\beta}^{\alpha}, \\
\left(\sigma_{\mu} \cdot \bar{\sigma}_{v}+\sigma_{v} \cdot \bar{\sigma}_{\mu}\right)_{\alpha}^{\beta}=-2 \eta_{\mu v} \delta_{\alpha}{ }^{\beta}, \\
\left(\bar{\sigma}_{\mu} \cdot \sigma_{v}+\bar{\sigma}_{v} \cdot \sigma_{\mu}\right)_{\dot{\beta}}^{\dot{\alpha}}=-2 \eta_{\mu v} \delta_{\dot{\beta}}^{\dot{\alpha}}, \\
\operatorname{Tr}\left(\sigma_{\mu} \cdot \bar{\sigma}_{v}\right)=\sigma_{\mu \alpha \dot{\alpha}} \bar{\sigma}_{v}{ }^{\dot{\alpha} \alpha}=-2 \eta_{\mu v},
\end{gathered}
$$

and $\sigma^{\lambda} \cdot \bar{\sigma}^{\mu} \cdot \sigma^{v}=\eta^{\lambda v} \sigma^{\mu}-\eta^{\lambda \mu} \sigma^{v}-\eta^{\mu v} \sigma^{\lambda}-i \varepsilon_{0123} \varepsilon^{\lambda \mu \nu \rho} \sigma_{\rho}$.

The factor $\varepsilon_{0123}= \pm 1$ was included to allow for ready use of both conventions for the four-dimensional epsilon tensor.

We will briefly recall below that pulling spinor indices with the $2^{\text {nd }}$ order epsilon spinors is motivated by the fact that this yields Lorentz invariant spinor products

$$
\psi \cdot \chi \equiv \psi^{\alpha} \chi_{\alpha}=\varepsilon^{\alpha \beta} \psi_{\beta} \chi_{\alpha}=-\psi_{\alpha} \chi^{\alpha}=\chi \cdot \psi,
$$

where the anti-commutation property of spinors was used. Conjugation also implies re-ordering of spinor quantities, such that conjugation of (9) yields

$$
\bar{\chi} \cdot \bar{\psi} \equiv \bar{\chi}_{\dot{\alpha}} \bar{\psi}^{\dot{\alpha}}=\bar{\chi}_{\dot{\alpha}} \varepsilon^{\dot{\alpha} \dot{\beta}} \bar{\psi}_{\dot{\beta}}=\bar{\psi} \cdot \bar{\chi} .
$$

The vector representation matrices of the Lorentz algebra,

$$
\left(L_{\mu v}\right)_{\rho}^{\sigma}=\eta_{\mu \rho} \eta_{v}{ }^{\sigma}-\eta_{\mu}{ }^{\sigma} \eta_{v \rho}
$$

appear as structure constants in the Poincaré algebra. The spinor representations of a proper orthochronous Lorentz transformation

$$
\Lambda=\exp \left(\frac{i}{2} \omega^{\mu v} M_{\mu v}\right)=\exp \left(\frac{1}{2} \omega^{\mu v} L_{\mu v}\right)
$$

are given by

$$
U(\Lambda)=\exp \left(\frac{i}{2} \omega^{\mu v} S_{\mu v}\right)
$$

and

with generators

$$
\tilde{U}(\Lambda)=\exp \left(\frac{i}{2} \omega^{\mu v} \bar{S}_{\mu v}\right),
$$

$$
\begin{aligned}
& \left(S_{\mu v}\right)_{\alpha}^{\beta}=\frac{i}{4}\left(\sigma_{\mu} \cdot \bar{\sigma}_{v}-\sigma_{v} \cdot \bar{\sigma}_{\mu}\right)_{\alpha}^{\beta}, \\
& \left(\bar{S}_{\mu v}\right)_{\dot{\beta}}^{\dot{\alpha}}=\frac{i}{4}\left(\bar{\sigma}_{\mu} \cdot \sigma_{v}-\bar{\sigma}_{v} \cdot \sigma_{\mu}\right)_{\dot{\beta}}^{\dot{\alpha}} .
\end{aligned}
$$


The relations

$$
\begin{aligned}
& \operatorname{Tr}\left(S_{\mu v} \cdot S_{\rho \sigma}\right)=\frac{1}{2}\left(\eta_{\mu \rho} \eta_{v \sigma}-\eta_{\mu \sigma} \eta_{\nu \rho}-i \varepsilon_{0123} \varepsilon_{\mu \nu \rho \sigma}\right), \\
& \operatorname{Tr}\left(\bar{S}_{\mu \nu} \cdot \bar{S}_{\rho \sigma}\right)=\frac{1}{2}\left(\eta_{\mu \rho} \eta_{v \sigma}-\eta_{\mu \sigma} \eta_{\nu \rho}+i \varepsilon_{0123} \varepsilon_{\mu v \rho \sigma}\right) .
\end{aligned}
$$

are used in the derivation of supersymmetric Maxwell or Yang-Mills actions.

The spinor products $(9,10)$ are invariant because the matrices $U(\Lambda)$ and $\tilde{U}(\Lambda)$ are $S L(2, \mathbb{C})$ matrices,

$$
\begin{gathered}
\varepsilon^{\alpha \beta} U(\Lambda)_{\alpha}^{\gamma} U(\Lambda)_{\beta}{ }^{\delta}=\varepsilon^{\gamma \delta}, \\
\varepsilon_{\dot{\alpha} \dot{\beta}} \tilde{U}(\Lambda)_{\dot{\gamma}}^{\dot{\alpha}} \tilde{U}(\Lambda)_{\dot{\delta}}^{\dot{\beta}}=\varepsilon_{\dot{\gamma} \dot{\delta}} .
\end{gathered}
$$

Stated differently, the epsilon spinors are Lorentz invariant.

We can now write down the super-Poincaré algebra in the form

$$
\begin{gathered}
{\left[M_{\mu v}, M_{\rho \sigma}\right]=i\left(L_{\mu \nu}\right)_{\rho}^{\lambda} M_{\lambda \sigma}+i\left(L_{\mu \nu}\right)_{\sigma}^{\lambda} M_{\rho \lambda},} \\
{\left[M_{\mu \nu}, p_{\rho}\right]=i\left(L_{\mu \nu}\right)_{\rho}^{\lambda} p_{\lambda},} \\
{\left[M_{\mu \nu}, Q_{\alpha}\right]=-\left(S_{\mu \nu}\right)_{\alpha}^{\beta} Q_{\beta},} \\
{\left[M_{\mu v}, \bar{Q}^{\dot{\alpha}}\right]=-\left(\bar{S}_{\mu \nu}\right)_{\dot{\beta}}^{\dot{\alpha}} \bar{Q}^{\dot{\beta}},} \\
\left\{Q_{\alpha}, \bar{Q}_{\dot{\alpha}}\right\}=2 p_{\alpha \dot{\alpha}}=2 p_{\mu} \sigma_{\alpha \dot{\alpha}}^{\mu}, \\
\left\{Q_{\alpha}, Q_{\beta}\right\}=0, \quad\left\{\bar{Q}_{\dot{\alpha}}, \bar{Q}_{\dot{\beta}}\right\}=0, \\
{\left[p_{\mu}, Q_{\alpha}\right]=0, \quad\left[p_{\mu}, \bar{Q}_{\dot{\alpha}}\right]=0 .}
\end{gathered}
$$

The $S L(2, \mathbb{C})$ property (13) reads in first order

$$
\left(\bar{S}_{\mu v}\right)_{\dot{\gamma} \dot{\delta}}=\left(\bar{S}_{\mu v}\right)_{\dot{\delta} \dot{\gamma}}
$$

and implies that Equation (14) can also be written as

$$
\left[M_{\mu v}, \bar{Q}_{\dot{\alpha}}\right]=\bar{Q}_{\dot{\beta}}\left(\bar{S}_{\mu v}\right)_{\dot{\alpha}}^{\dot{\beta}} \text {. }
$$

The super-Poincaré algebra satisfies all the pertinent super-Jacobi identities as a consequence of the representation properties of the vector and spinor representations of the Lorentz algebra. The particular super-Jacobi identity

$$
\left[M_{\mu v},\left\{Q_{\alpha}, \bar{Q}_{\dot{\alpha}}\right\}\right]=\left\{\left[M_{\mu v} Q_{\alpha}\right], \bar{Q}_{\dot{\alpha}}\right\}+\left\{\left[M_{\mu v} \bar{Q}_{\dot{\alpha}}\right], Q_{\alpha}\right\}
$$

holds as a consequence of the fact that the Pauli matrices have the same form in every inertial frame,

$$
\begin{aligned}
\left(L_{\mu v}\right)_{\kappa}^{\lambda} \sigma_{\alpha \dot{\alpha}}^{\kappa} & =\eta_{\mu}^{\lambda} \sigma_{v \alpha \dot{\alpha}}-\eta_{v}{ }^{\lambda} \sigma_{\mu \alpha \dot{\alpha}} \\
& =\frac{1}{4}\left(\sigma_{\mu} \cdot \bar{\sigma}_{v}-\sigma_{v} \cdot \bar{\sigma}_{\mu}\right)_{\alpha}^{\beta} \sigma_{\beta \dot{\alpha}}^{\lambda} \\
& -\sigma_{\alpha \dot{\beta}}^{\lambda} \frac{1}{4}\left(\bar{\sigma}_{\mu} \cdot \sigma_{v}-\bar{\sigma}_{v} \cdot \sigma_{\mu}\right)_{\dot{\alpha}}^{\dot{\beta}} .
\end{aligned}
$$

This can be verified from Equations $(5,6)$ by commuting the $\sigma^{\lambda}$ matrices into the middle positions in the products on the right hand side. It can also be verified as a direct consequence of Equation (8).

\section{3. $N=1$ Superspace}

The Poincaré algebra is realized on spacetime coordinates $x^{\mu}$ through derivative operators

$$
M_{\mu \nu}=-i\left(x_{\mu} \partial_{v}-x_{v} \partial_{\mu}\right), \quad p_{\mu}=-i \partial_{\mu}
$$

In a nutshell, superspace is based on the observation that this construction can be extended to the super-Poincaré algebra by supplementing Minkowski spacetime with fermionic coordinates $\theta^{\alpha}$ and $\bar{\theta}^{\dot{\alpha}}$ and corresponding fermionic derivatives

$$
\partial_{\alpha} \theta^{\beta}=\delta_{\alpha}{ }^{\beta}, \quad \bar{\partial}_{\dot{\alpha}} \bar{\theta}^{\dot{\beta}}=\delta_{\dot{\alpha}}^{\dot{\beta}} .
$$

The super-Poincaré algebra is then realized on the superspace coordinates $\left(x^{\mu}, \theta^{\alpha}, \bar{\theta}^{\dot{\alpha}}\right)$ by amending the representations (16) of the bosonic operators with the realizations

$$
Q_{\alpha}=-i \partial_{\alpha}-\sigma_{\alpha \dot{\alpha}}^{\mu} \bar{\theta}^{\dot{\alpha}} \partial_{\mu}, \quad \bar{Q}_{\dot{\alpha}}=i \bar{\partial}_{\dot{\alpha}}+\theta^{\alpha} \sigma_{\alpha \dot{\alpha}}^{\mu} \partial_{\mu},
$$

for the fermionic operators and complementing the Lorentz generators to include the action on $Q_{\alpha}$ and $\bar{Q}_{\dot{\alpha}}$,

$$
M_{\mu v}=-i\left(x_{\mu} \partial_{v}-x_{v} \partial_{\mu}\right)+\theta^{\alpha}\left(S_{\mu v}\right)_{\alpha}^{\beta} \partial_{\beta}-\left(\bar{S}_{\mu v}\right)_{\dot{\alpha}}^{\dot{\beta}} \bar{\theta}^{\dot{\alpha}} \bar{\partial}_{\dot{\beta}} .
$$

A superfield $V(x, \theta, \bar{\theta})$ maps a spacetime point $x$ into the algebra over $\mathbb{C}$ which is generated by the five elements $(1, \theta, \bar{\theta})$, subject to the relations $(35,36)$ in the Appendix A.2. Note that this definition explicitly refers to the fermionic arguments of $V$. Supertranslations are based on the concept that there are infinitely many incarnations of the four fermionic generators $\theta^{\alpha}$ and $\bar{\theta}^{\dot{\alpha}}$ and that we can freely move between these infinitely many copies of the same algebra. In particular, if $(1, \theta, \bar{\theta})$ and $(1, \zeta, \bar{\zeta})$ generate the same algebra, we require that $(1, \theta+\zeta, \bar{\theta}+\bar{\zeta})$ also generates the same algebra. This requirement is equivalent to anti-commutation properties

$$
\theta_{\alpha} \zeta_{\beta}=-\zeta_{\beta} \theta_{\alpha}, \quad \bar{\theta}_{\dot{\alpha}} \zeta_{\beta}=-\zeta_{\beta} \bar{\theta}_{\dot{\alpha}},
$$

and the corresponding conjugate equations, i.e. the algebra generated by $(1, \theta, \bar{\theta})$ is a subalgebra of a corresponding infinite-dimensional algebra.

The relations in Appendix A.2 imply that the expansion of every superfield with respect to the fermionic 
elements $\theta^{\alpha}$ and $\bar{\theta}^{\dot{\alpha}}$ can be written in terms of four scalars $\phi(x), M(x), N(x), D(x)$, four Weyl fermions $\psi(x), \chi(x), \kappa(x), \lambda(x)$, and a vector field $A_{\mu}(x)$, $V(x, \theta, \bar{\theta})=\phi(x)+\theta \cdot \psi(x)+\bar{\chi}(x) \cdot \bar{\theta}+\theta \cdot \sigma^{\mu} \cdot \bar{\theta} A_{\mu}(x)$ $+\theta^{2} M(x)+\bar{\theta}^{2} N(x)+\bar{\theta}^{2} \theta \cdot \kappa(x)+\theta^{2} \bar{\lambda}(x) \cdot \bar{\theta}+\theta^{2} \bar{\theta}^{2} D(x)$.

The commutation relations

$$
\begin{aligned}
& i\left[\zeta \cdot Q, \theta^{\alpha}\right]=\zeta^{\alpha}, \quad i\left[\bar{Q} \cdot \bar{\zeta}, \bar{\theta}^{\dot{\alpha}}\right]=\bar{\zeta}^{\dot{\alpha}}, \\
& i\left[\zeta \cdot Q+\bar{Q} \cdot \bar{\zeta}, x^{\mu}\right]=-i\left(\zeta \cdot \sigma^{\mu} \cdot \bar{\theta}-\theta \cdot \sigma^{\mu} \cdot \bar{\zeta}\right)
\end{aligned}
$$

imply for unitary supertranslations

$$
\begin{aligned}
& \left|x^{\prime}, \theta^{\prime}, \bar{\theta}^{\prime}\right\rangle=\exp [i(\zeta \cdot Q+\bar{Q} \cdot \bar{\zeta})]|x, \theta, \bar{\theta}\rangle \\
& =|x+i(\zeta \cdot \sigma \cdot \bar{\theta}-\theta \cdot \sigma \cdot \bar{\zeta}), \theta-\zeta, \bar{\theta}-\bar{\zeta}\rangle,
\end{aligned}
$$

and therefore

$$
\begin{aligned}
& V^{\prime}(x, \theta, \bar{\theta})=\langle x, \theta, \bar{\theta}|\exp [i(\zeta \cdot Q+\bar{Q} \cdot \bar{\zeta})]| V\rangle \\
& =V(x-i(\zeta \cdot \sigma \cdot \bar{\theta}-\theta \cdot \sigma \cdot \bar{\zeta}), \theta+\zeta, \bar{\theta}+\bar{\zeta}) .
\end{aligned}
$$

We can calculate the transformation properties of the component fields by comparing

$$
\begin{aligned}
& V^{\prime}(x, \theta, \bar{\theta})=\phi^{\prime}(x)+\theta \cdot \psi^{\prime}(x)+\bar{\chi}^{\prime}(x) \cdot \bar{\theta}+\theta \cdot \sigma^{\mu} \cdot \bar{\theta} A_{\mu}^{\prime}(x) \\
& +\theta^{2} M^{\prime}(x)+\bar{\theta}^{2} N^{\prime}(x)+\bar{\theta}^{2} \theta \cdot \kappa^{\prime}(x)+\theta^{2} \bar{\lambda}^{\prime}(x) \cdot \bar{\theta} \\
& +\theta^{2} \bar{\theta}^{2} D^{\prime}(x)
\end{aligned}
$$

with the expansion of the right hand side of (19) with respect to the fermionic variables $\theta^{\alpha}$ and $\bar{\theta}^{\dot{\alpha}}$.

\section{Supertranslations of the General Linear Multiplet}

Equation (19) implies in particular that supertranslations shift the argument $x$ of component fields to

$$
X^{\mu}=x^{\mu}-i\left(\zeta \cdot \sigma^{\mu} \cdot \bar{\theta}-\theta \cdot \sigma^{\mu} \cdot \bar{\zeta}\right) .
$$

We can calculate the transformation properties of the components of the supermultiplet $V$ to all orders in the translation parameters $\zeta, \bar{\zeta}$, by expanding the right hand side

$$
\begin{aligned}
& V(X, \theta+\zeta, \bar{\theta}+\bar{\zeta})=\phi(x-i(\zeta \cdot \sigma \cdot \bar{\theta}-\theta \cdot \sigma \cdot \bar{\zeta})) \\
& +(\theta+\zeta) \cdot \psi(x-i(\zeta \cdot \sigma \cdot \bar{\theta}-\theta \cdot \sigma \cdot \bar{\zeta}))+\ldots
\end{aligned}
$$

of Equation (19) to all orders in $\theta$ and $\bar{\theta}$.

The first step requires the expansion of the component fields with respect to the coordinate shifts

$$
\Delta x^{\mu}=-i\left(\zeta \cdot \sigma^{\mu} \cdot \bar{\theta}-\theta \cdot \sigma^{\mu} \cdot \bar{\zeta}\right),
$$

e.g.

$$
\begin{aligned}
& \phi(X)=\phi(x)-i\left(\zeta \cdot \sigma^{\mu} \cdot \bar{\theta}-\theta \cdot \sigma^{\mu} \cdot \bar{\zeta}\right) \partial_{\mu} \phi(x) \\
& -\frac{1}{2}\left(\zeta \cdot \sigma^{\mu} \cdot \bar{\theta}-\theta \cdot \sigma^{\mu} \cdot \bar{\zeta}\right)\left(\zeta \cdot \sigma^{v} \cdot \bar{\theta}-\theta \cdot \sigma^{\nu} \cdot \bar{\zeta}\right) \partial_{\mu} \partial_{v} \phi(x) \\
& +\frac{i}{6}\left(\zeta \cdot \sigma^{\lambda} \cdot \bar{\theta}-\theta \cdot \sigma^{\lambda} \cdot \bar{\zeta}\right)\left(\zeta \cdot \sigma^{\mu} \cdot \bar{\theta}-\theta \cdot \sigma^{\mu} \cdot \bar{\zeta}\right) \\
& \times\left(\zeta \cdot \sigma^{v} \cdot \bar{\theta}-\theta \cdot \sigma^{v} \cdot \bar{\zeta}\right) \partial_{\lambda} \partial_{\mu} \partial_{v} \phi(x) \\
& +\frac{1}{24}\left(\zeta \cdot \sigma^{\kappa} \cdot \bar{\theta}-\theta \cdot \sigma^{\kappa} \cdot \bar{\zeta}\right)\left(\zeta \cdot \sigma^{\lambda} \cdot \bar{\theta}-\theta \cdot \sigma^{\lambda} \cdot \bar{\zeta}\right) \\
& \times\left(\zeta \cdot \sigma^{\mu} \cdot \bar{\theta}-\theta \cdot \sigma^{\mu} \cdot \bar{\zeta}\right)\left(\zeta \cdot \sigma^{v} \cdot \bar{\theta}-\theta \cdot \sigma^{v} \cdot \bar{\zeta}\right) \partial_{\kappa} \partial_{\lambda} \partial_{\mu} \partial_{v} \phi(x) \\
& =\phi(x)-i\left(\zeta \cdot \sigma^{\mu} \cdot \bar{\theta}-\theta \cdot \sigma^{\mu} \cdot \bar{\zeta}\right) \partial_{\mu} \phi(x) \\
& +\frac{1}{4}\left(\zeta^{2} \bar{\theta}^{2}+\theta^{2} \bar{\zeta}^{2}+2 \zeta \cdot \sigma_{\mu} \cdot \bar{\zeta} \theta \cdot \sigma^{\mu} \cdot \bar{\theta}\right) \partial^{2} \phi(x) \\
& -\frac{1}{2}\left(\zeta \cdot \sigma^{\nu} \cdot \bar{\zeta} \theta \cdot \sigma^{\mu} \cdot \bar{\theta}+\zeta \cdot \sigma^{\mu} \cdot \bar{\zeta} \theta \cdot \sigma^{\nu} \cdot \bar{\theta}\right) \partial_{\mu} \partial_{v} \phi(x) \\
& +\frac{i}{4}\left(\zeta^{2} \bar{\theta}^{2} \theta \cdot \sigma^{\mu} \cdot \bar{\zeta}-\theta^{2} \bar{\zeta}^{2} \zeta \cdot \sigma^{\mu} \cdot \bar{\theta}\right) \partial_{\mu} \partial^{2} \phi(x) \\
& +\frac{1}{16} \theta^{2} \bar{\theta}^{2} \zeta^{2} \bar{\zeta}^{2} \partial^{2} \partial^{2} \phi(x),
\end{aligned}
$$

and corresponding expansions for combinations of the other eight component fields with various factors, which are different in each case due to the presence of fermionic variables in the extra factors. Altogether, this includes 35 more relations, e.g.

$$
\begin{aligned}
& \theta \cdot \psi(X)=\theta \cdot \psi(x)-\frac{i}{2} \theta \cdot \sigma^{\mu} \cdot \bar{\theta} \zeta \cdot \sigma^{v} \cdot \bar{\sigma}_{\mu} \cdot \partial_{v} \psi(x) \\
& +\frac{i}{2} \theta^{2} \bar{\zeta} \cdot \bar{\sigma}^{\mu} \cdot \partial_{\mu} \psi(x)+\frac{1}{4} \zeta^{2} \bar{\theta}^{2} \theta \cdot \partial^{2} \psi(x) \\
& +\frac{1}{4} \theta^{2} \zeta \cdot \sigma_{\mu} \cdot \overline{\zeta \theta} \cdot \bar{\sigma}^{\mu} \cdot \partial^{2} \psi(x) \\
& -\frac{1}{4} \theta^{2}\left(\zeta \cdot \sigma^{\mu} \cdot \overline{\zeta \theta} \cdot \bar{\sigma}^{v}+\zeta \cdot \sigma^{v} \cdot \overline{\zeta \theta} \cdot \bar{\sigma}^{\mu}\right) \cdot \partial_{\mu} \partial_{v} \psi(x) \\
& -\frac{i}{8} \theta^{2} \bar{\theta}^{2} \zeta^{2} \partial_{\mu} \partial^{2} \psi(x) \cdot \sigma^{\mu} \cdot \bar{\zeta} .
\end{aligned}
$$

Substitution of all the expansions in terms of standard words in the Grassmann variables into Equation (19) yields the full supertranslation properties of the component fields, which are reported in Equations (21-29). The transformation equations of the component fields are organized by contributions from the nine component fields $\phi(x), \psi(x), \bar{\chi}(x), A_{\mu}(x), M(x), N(x), \kappa(x)$,

$\bar{\lambda}(x)$, and $D(x)$, instead of organization by expansion in the supertranslation parameters $\zeta$ and $\bar{\zeta}$. In this way, supertranslations act on the component fields like matrices which have Grassmann valued differential 
operators as entries. The reader can easily re-organize the transformation equations in terms of supertranslation parameters. The supertranslation equations are

$$
\begin{aligned}
& \phi^{\prime}(x)=\phi(x)+\zeta \cdot \psi(x)+\bar{\chi}(x) \cdot \bar{\zeta} \\
& +\zeta \cdot \sigma^{\mu} \cdot \bar{\zeta} A_{\mu}(x)+\zeta^{2} M(x)+\bar{\zeta}^{2} N(x) \\
& +\bar{\zeta}^{2} \zeta \cdot \kappa(x)+\zeta^{2} \bar{\lambda}(x) \cdot \bar{\zeta}+\zeta^{2} \bar{\zeta}^{2} D(x), \\
& \psi^{\prime}(x)=i \sigma^{\mu} \cdot \bar{\zeta} \partial_{\mu} \phi(x)+\psi(x) \\
& +\frac{i}{2} \sigma^{\mu} \cdot \bar{\sigma}_{v} \cdot \partial_{\mu} \psi(x) \zeta \cdot \sigma^{v} \cdot \bar{\zeta} \\
& -\frac{i}{2} \bar{\zeta}^{2} \sigma^{\mu} \cdot \partial_{\mu} \bar{\chi}(x)+\sigma^{\mu} \cdot \bar{\zeta} A_{\mu}(x) \\
& +\frac{i}{2} \sigma^{v} \cdot \bar{\sigma}^{\mu} \cdot \zeta \bar{\zeta}^{2} \partial_{v} A_{\mu}(x)+2 \zeta M(x) \\
& +i \zeta^{2} \sigma^{\mu} \cdot \bar{\zeta} \partial_{\mu} M(x)+\kappa(x) \bar{\zeta}^{2} \\
& -\sigma_{\mu} \cdot \bar{\lambda}(x) \zeta \cdot \sigma^{\mu} \cdot \bar{\zeta}-\frac{i}{2} \zeta^{2} \bar{\zeta}^{2} \sigma^{\mu} \cdot \partial_{\mu} \bar{\lambda}(x) \\
& +2 \zeta \bar{\zeta}^{2} D(x) \text {, } \\
& \bar{\chi}^{\prime}(x)=-i \partial_{\mu} \phi(x) \zeta \cdot \sigma^{\mu}+\frac{i}{2} \zeta^{2} \partial_{\mu} \psi(x) \cdot \sigma^{\mu} \\
& +\bar{\chi}(x)-\frac{i}{2} \zeta \cdot \sigma^{v} \cdot \bar{\zeta} \partial_{\mu} \bar{\chi}(x) \cdot \bar{\sigma}_{v} \cdot \sigma^{\mu} \\
& +A_{\mu}(x) \zeta \cdot \sigma^{\mu}-\frac{i}{2} \zeta^{2} \partial_{v} A_{\mu}(x) \bar{\zeta} \cdot \bar{\sigma}^{\mu} \cdot \sigma^{v} \\
& +2 \bar{\zeta} N(x)-i \bar{\zeta}^{2} \partial_{\mu} N(x) \zeta \cdot \sigma^{\mu} \\
& -\zeta \cdot \sigma^{\mu} \cdot \bar{\zeta} \kappa(x) \cdot \sigma_{\mu}+\frac{i}{2} \zeta^{2} \bar{\zeta}^{2} \partial_{\mu} \kappa(x) \cdot \sigma^{\mu} \\
& +\zeta^{2} \bar{\lambda}(x)+2 \zeta^{2} \bar{\zeta} D(x) \text {, } \\
& M^{\prime}(x)=\frac{1}{4} \bar{\zeta}^{2} \partial^{2} \phi(x)-\frac{i}{2} \partial_{\mu} \psi(x) \cdot \sigma^{\mu} \cdot \bar{\zeta} \\
& +\frac{1}{4} \bar{\zeta}^{2} \zeta \cdot \partial^{2} \psi(x)-\frac{i}{2} \bar{\zeta}^{2} \partial_{\mu} A^{\mu}(x)+M(x) \\
& -i \zeta \cdot \sigma^{\mu} \cdot \bar{\zeta} \partial_{\mu} M(x)+\frac{1}{4} \zeta^{2} \bar{\zeta}^{2} \partial^{2} M(x) \\
& +\bar{\zeta} \cdot \bar{\lambda}(x)+\frac{i}{2} \bar{\zeta}^{2} \zeta \cdot \sigma^{\mu} \cdot \partial_{\mu} \bar{\lambda}(x)+\bar{\zeta}^{2} D(x), \\
& N^{\prime}(x)=\frac{1}{4} \zeta^{2} \partial^{2} \phi(x)+\frac{i}{2} \zeta \cdot \sigma^{\mu} \cdot \partial_{\mu} \bar{\chi}(x) \\
& +\frac{1}{4} \zeta^{2} \bar{\zeta} \cdot \partial^{2} \bar{\chi}(x)+\frac{i}{2} \zeta^{2} \partial_{\mu} A^{\mu}(x)+N(x) \\
& +i \zeta \cdot \sigma^{\mu} \cdot \bar{\zeta} \partial_{\mu} N(x)+\frac{1}{4} \zeta^{2} \bar{\zeta}^{2} \partial^{2} N(x) \\
& +\zeta \cdot \kappa(x)-\frac{i}{2} \zeta^{2} \partial_{\mu} \kappa(x) \cdot \sigma^{\mu} \cdot \bar{\zeta}+\zeta^{2} D(x),
\end{aligned}
$$$$
-\frac{1}{4} \zeta^{2} \bar{\zeta}^{2}\left(\partial^{2} A_{\mu}(x)-2 \partial_{\mu} \partial_{v} A^{v}(x)\right)
$$$$
+i \zeta^{2} \partial_{\mu} M(x)-i \bar{\zeta}^{2} \partial_{\mu} N(x)-\kappa(x) \cdot \sigma_{\mu} \cdot \bar{\zeta}
$$$$
-i \bar{\zeta}^{2} \zeta \cdot \partial_{\mu} \kappa(x)-\frac{i}{2} \bar{\zeta}^{2} \zeta \cdot \sigma^{\nu} \cdot \bar{\sigma}_{\mu} \cdot \partial_{\nu} \kappa(x)
$$$$
-\zeta \cdot \sigma_{\mu} \cdot \bar{\lambda}(x)+i \zeta^{2} \bar{\zeta} \cdot \partial_{\mu} \bar{\lambda}(x)
$$$$
+\frac{i}{2} \zeta^{2} \partial_{v} \bar{\lambda}(x) \cdot \bar{\sigma}_{\mu} \cdot \sigma^{v} \cdot \bar{\zeta}-2 \zeta \cdot \sigma_{\mu} \cdot \bar{\zeta} D(x) \text {. }
$$

For conversion of the last equation into standard words in the Grassmann variables $\zeta$ and $\bar{\zeta}$, note that from Equation (8)

$$
\begin{aligned}
& \frac{i}{2} \zeta \cdot \sigma^{\lambda} \cdot \bar{\sigma}_{\mu} \cdot \sigma^{v} \cdot \bar{\zeta} F_{v \lambda}(x)=\frac{1}{2} \varepsilon_{0123} \varepsilon_{\mu{ }_{\mu}{ }_{\rho} \zeta} \zeta \cdot \sigma^{\rho} \cdot \bar{\zeta} F_{v \lambda}(x) \\
& =\varepsilon_{0123} \tilde{F}_{\mu \rho}(x) \zeta \cdot \sigma^{\rho} \cdot \bar{\zeta} .
\end{aligned}
$$

The remaining transformation equations are

$$
\begin{aligned}
& \kappa^{\prime}(x)=\frac{i}{4} \zeta^{2} \sigma^{\mu} \cdot \bar{\zeta} \partial_{\mu} \partial^{2} \phi(x)+\frac{1}{4} \zeta^{2} \partial^{2} \psi(x) \\
& -\frac{1}{4}\left(\zeta \cdot \sigma_{\mu} \cdot \bar{\zeta}\right) \sigma^{\mu} \cdot \partial^{2} \bar{\chi}(x) \\
& +\frac{1}{2}\left(\zeta \cdot \sigma^{\mu} \cdot \bar{\zeta}\right) \sigma^{v} \cdot \partial_{\mu} \partial_{v} \bar{\chi}(x) \\
& -\frac{i}{8} \zeta^{2} \bar{\zeta}^{2} \sigma^{\mu} \cdot \partial_{\mu} \partial^{2} \bar{\chi}(x)-\frac{i}{2} \sigma^{\mu} \cdot \bar{\sigma}^{v} \cdot \zeta \partial_{v} A_{\mu}(x) \\
& +\frac{1}{4} \zeta^{2} \sigma^{\mu} \cdot \bar{\zeta}\left(\partial^{2} A_{\mu}(x)-2 \partial_{\mu} \partial_{v} A^{v}(x)\right) \\
& +i \sigma^{\mu} \cdot \bar{\zeta} \partial_{\mu} N(x)+\frac{1}{2} \zeta \bar{\zeta}^{2} \partial^{2} N(x)+\kappa(x) \\
& +\frac{i}{2}\left(\zeta \cdot \sigma^{v} \cdot \bar{\zeta}\right) \sigma^{\mu} \cdot \bar{\sigma}_{v} \cdot \partial_{\mu} \kappa(x) \\
& +i\left(\zeta \cdot \sigma^{\mu} \cdot \bar{\zeta}\right) \partial_{\mu} \kappa(x)-\frac{i}{2} \zeta^{2} \sigma^{\mu} \cdot \partial_{\mu} \bar{\lambda}(x) \\
& +2 \zeta D(x),
\end{aligned}
$$




$$
\begin{aligned}
& \bar{\lambda}^{\prime}(x)=-\frac{i}{4} \bar{\zeta}^{2} \partial_{\mu} \partial^{2} \phi(x) \zeta \cdot \sigma^{\mu} \\
& -\frac{1}{4}\left(\zeta \cdot \sigma^{\mu} \cdot \bar{\zeta}\right)\left(\partial^{2} \psi(x) \cdot \sigma_{\mu}-2 \partial_{\mu} \partial_{\nu} \psi(x) \cdot \sigma^{v}\right) \\
& +\frac{i}{8} \zeta^{2} \bar{\zeta}^{2} \partial_{\mu} \partial^{2} \psi(x) \cdot \sigma^{\mu}+\frac{1}{4} \bar{\zeta}^{2} \partial^{2} \bar{\chi}(x) \\
& +\frac{i}{2} \partial_{v} A_{\mu}(x) \bar{\zeta} \cdot \bar{\sigma}^{v} \cdot \sigma^{\mu}+\frac{1}{4} \bar{\zeta}^{2} \partial^{2} A_{\mu}(x) \zeta \cdot \sigma^{\mu} \\
& -\frac{1}{2} \bar{\zeta}^{2} \zeta \cdot \sigma^{\mu} \partial_{\mu} \partial_{v} A^{v}(x)-i \partial_{\mu} M(x) \zeta \cdot \sigma^{\mu} \\
& +\frac{1}{2} \zeta^{2} \bar{\zeta} \partial^{2} M(x)+\frac{i}{2} \bar{\zeta}^{2} \partial_{\mu} \kappa(x) \cdot \sigma^{\mu}+\bar{\lambda}(x) \\
& -\frac{i}{2}\left(\zeta \cdot \sigma^{v} \cdot \bar{\zeta}\right) \partial_{\mu} \bar{\lambda}(x) \cdot \bar{\sigma}_{v} \cdot \sigma^{\mu} \\
& -i\left(\zeta \cdot \sigma^{\mu} \cdot \bar{\zeta}\right) \partial_{\mu} \bar{\lambda}(x)+2 \bar{\zeta} D(x) \\
& D^{\prime}(x)=\frac{1}{16} \zeta^{2} \bar{\zeta}^{2} \partial^{2} \partial^{2} \phi(x) \\
& -\frac{i}{8} \zeta^{2} \partial_{\mu} \partial^{2} \psi(x) \cdot \sigma^{\mu} \cdot \bar{\zeta}+\frac{i}{8} \bar{\zeta}^{2} \zeta \cdot \sigma^{\mu} \cdot \partial_{\mu} \partial^{2} \bar{\chi}(x) \\
& -\frac{1}{4} \zeta \cdot \sigma^{\mu} \cdot \bar{\zeta}\left(\partial^{2} A_{\mu}(x)-2 \partial_{\mu} \partial_{v} A^{v}(x)\right) \\
& +\frac{1}{4} \zeta^{2} \partial^{2} M(x)+\frac{1}{4} \bar{\zeta}^{2} \partial^{2} N(x)-\frac{i}{2} \partial_{\mu} \kappa(x) \cdot \sigma^{\mu} \cdot \bar{\zeta} \\
& +\frac{i}{2} \zeta \cdot \sigma^{\mu} \cdot \partial_{\mu} \bar{\lambda}(x)+D(x) \text {. }
\end{aligned}
$$

These transformation laws are compatible with the reality constraints $V(x, \theta, \bar{\theta})=V^{\dagger}(x, \theta, \bar{\theta})$ which define the vector multiplet,

$$
\begin{gathered}
\phi(x)=\phi^{\dagger}(x), \psi(x)=\chi(x), A_{\mu}(x)=A_{\mu}^{\dagger}(x), \\
M(x)=N^{\dagger}(x), \kappa(x)=\lambda(x), D(x)=D^{\dagger}(x) .
\end{gathered}
$$

\section{Supertranslations of the Chiral Multiplet}

Besides the superderivatives (18) one can also define supercovariant derivatives $[18,19]$

$$
D_{\alpha}=-i \partial_{\alpha}+\sigma_{\alpha \dot{\alpha}}^{\mu} \bar{\theta}^{\dot{\alpha}} \partial_{\mu}, \quad \bar{D}_{\dot{\alpha}}=i \bar{\partial}_{\dot{\alpha}}-\theta^{\alpha} \sigma_{\alpha \dot{\alpha}}^{\mu} \partial_{\mu},
$$

such that

$$
\left\{D_{\alpha}, D_{\beta}\right\}=0,\left\{\bar{D}_{\dot{\alpha}}, \bar{D}_{\dot{\beta}}\right\}=0,\left\{D_{\alpha}, \bar{D}_{\dot{\alpha}}\right\}=2 i \sigma_{\alpha \dot{\alpha}}^{\mu} \partial_{\mu},
$$

and

$$
\left\{Q_{\alpha}, D_{\beta}\right\}=0,\left\{\bar{Q}_{\dot{\alpha}}, \bar{D}_{\dot{\beta}}\right\}=0,\left\{Q_{\alpha}, \bar{D}_{\dot{\alpha}}\right\}=0,\left\{\bar{Q}_{\dot{\alpha}}, D_{\alpha}\right\}=0 \text {. }
$$

The condition for chiral superfields

$$
\left(i \bar{\partial}_{\dot{\alpha}}-\theta^{\alpha} \sigma_{\alpha \dot{\alpha}}^{\mu} \partial_{\mu}\right) \Phi(x, \theta, \bar{\theta})=0
$$

is therefore invariant under supertranslations .

The basic solutions

$$
\bar{D}_{\dot{\alpha}} \theta^{\alpha}=0, \quad \bar{D}_{\dot{\alpha}}\left(x^{\mu}+i \theta \cdot \sigma^{\mu} \cdot \bar{\theta}\right)=0,
$$

imply [15]

$$
\begin{aligned}
& \Phi(x, \theta, \bar{\theta})=\Phi(x+i \theta \cdot \sigma \cdot \bar{\theta}, \bar{\theta})=\phi(x+i \theta \cdot \sigma \cdot \bar{\theta}) \\
& +\theta \cdot \psi(x+i \theta \cdot \sigma \cdot \bar{\theta})+\theta^{2} F(x+i \theta \cdot \sigma \cdot \bar{\theta}) .
\end{aligned}
$$

The relation

$$
-\left(\theta \cdot \sigma^{\mu} \cdot \bar{\theta}\right)\left(\theta \cdot \sigma^{\mu} \cdot \bar{\theta}\right) \partial_{\mu} \partial_{v}=\frac{1}{2} \theta^{2} \bar{\theta}^{2} \partial^{2}
$$

yields

$$
\begin{aligned}
& \Phi(x, \theta, \bar{\theta})=\phi(x)+i \theta \cdot \sigma^{\mu} \cdot \bar{\theta} \partial_{\mu} \phi(x) \\
& +\frac{1}{4} \theta^{2} \bar{\theta}^{2} \partial^{2} \phi(x)+\theta \cdot \psi(x) \\
& -\frac{i}{2} \theta^{2} \partial_{\mu} \psi(x) \cdot \sigma^{\mu} \cdot \bar{\theta}+\theta^{2} F(x) .
\end{aligned}
$$

The chiral superfield corresponds to the following substitutions in the general superfield $V$,

$$
\begin{gathered}
\bar{\chi}(x) \rightarrow 0, A_{\mu}(x) \rightarrow i \partial_{\mu} \phi(x), M(x) \rightarrow F(x), \\
N(x) \rightarrow 0, \kappa(x) \rightarrow 0, \bar{\lambda}(x) \rightarrow-\frac{i}{2} \partial_{\mu} \psi(x) \cdot \sigma^{\mu}, \\
D(x) \rightarrow \frac{1}{4} \partial^{2} \phi(x) .
\end{gathered}
$$

It is clear from the construction, but can also be checked explicitly that these constraints are compatible with the transformation laws (21-29) of the full linear multiplet.

We find the following supertranslations of the components of the chiral multiplet,

$$
\begin{aligned}
& \phi^{\prime}(x)=\phi(x)+i \zeta \cdot \sigma^{\mu} \cdot \bar{\zeta} \partial_{\mu} \phi(x)+\frac{1}{4} \zeta^{2} \bar{\zeta}^{2} \partial^{2} \phi(x) \\
& +\zeta \cdot \psi(x)-\frac{i}{2} \zeta^{2} \partial_{\mu} \psi(x) \cdot \sigma^{\mu} \cdot \bar{\zeta}+\zeta^{2} F(x), \\
& \psi^{\prime}(x)=2 i \sigma^{\mu} \cdot \bar{\zeta} \partial_{\mu} \phi(x)+\zeta^{2} \partial^{2} \phi(x)+\psi(x) \\
& +2\left(\zeta \cdot \sigma_{v} \cdot \bar{\zeta}\right) S^{\mu \nu} \cdot \partial_{\mu} \psi(x)-\frac{1}{4} \zeta^{2} \bar{\zeta}^{2} \partial^{2} \psi(x) \\
& +2 \zeta F(x)+i \zeta^{2} \sigma^{\mu} \cdot \bar{\zeta} \partial_{\mu} F(x), \\
& F^{\prime}(x)=\bar{\zeta}^{2} \partial^{2} \phi(x)-i \partial_{\mu} \psi(x) \cdot \sigma^{\mu} \cdot \bar{\zeta} \\
& +\frac{1}{2} \bar{\zeta}^{2} \zeta \cdot \partial^{2} \psi(x)+F(x) \\
& -i \zeta \cdot \sigma^{\mu} \cdot \bar{\zeta} \partial_{\mu} F(x)+\frac{1}{4} \zeta^{2} \bar{\zeta}^{2} \partial^{2} F(x) .
\end{aligned}
$$

Please note that this presentation does not involve the usual rescaling $\psi(x) \rightarrow \sqrt{2} \psi(x)$ of the spinor component of the chiral superfield, which is required for canonically normalized kinetic terms in supersymmetric Lagrangians. 


\section{Conclusions}

The supertranslation properties of the component fields of a general linear supermultiplet and of a chiral multiplet were reported to all orders in the translation parameters $\zeta$ and $\bar{\zeta}$ in (21-29) and (32-34), respectively. On the one hand, one can think of these results as explicit parametrizations of orbits of supertranslations in the space of component fields of a supersymmetric theory. On the other hand, one can consider the transformed fields as superfields in the variables $(x, \zeta, \bar{\zeta})$, because e.g.

$$
\begin{aligned}
& \psi_{\alpha}^{\prime}(x)=\psi_{\alpha}^{\prime}(x, \zeta, \bar{\zeta}) \\
& =\left[\frac{\partial}{\partial \theta^{\alpha}} V(x-i(\zeta \cdot \sigma \cdot \bar{\theta}-\theta \cdot \sigma \cdot \bar{\zeta}), \theta+\zeta, \bar{\theta}+\bar{\zeta})\right]_{\substack{\theta=0, \bar{\theta}=0}} \\
& =\left(i \sigma_{\alpha \dot{\alpha}}^{\mu} \bar{\zeta}^{\dot{\alpha}}+\frac{\partial}{\partial \zeta^{\alpha}}\right) V(x, \zeta, \bar{\zeta})=i D_{\alpha}^{(\zeta)} V(x, \zeta, \bar{\zeta}),
\end{aligned}
$$

and higher order derivatives with respect to the $\theta$ and $\bar{\theta}$ variables at $\theta=0, \bar{\theta}=0$ can also be expressed as supercovariant derivatives with respect to $\zeta$ and $\bar{\zeta}$. For example, the transformed vector field is

$$
\begin{aligned}
& A_{\alpha \dot{\alpha}}^{\prime}(x, \zeta, \bar{\zeta})=A_{\mu}^{\prime}(x, \zeta, \bar{\zeta}) \sigma_{\alpha \dot{\alpha}}^{\mu} \\
& =\left[\frac{\partial}{\partial \bar{\theta}^{\dot{\alpha}}} \frac{\partial}{\partial \theta^{\alpha}} V(x-i(\zeta \cdot \sigma \cdot \bar{\theta}-\theta \cdot \sigma \cdot \bar{\zeta}), \theta+\zeta, \bar{\theta}+\bar{\zeta})\right]_{\substack{\theta=0, \theta=0}} . \\
& \text { We have } \\
& \frac{\partial}{\partial \bar{\theta}^{\dot{\alpha}}} \frac{\partial}{\partial \theta^{\alpha}} V(x-i(\zeta \cdot \sigma \cdot \bar{\theta}-\theta \cdot \sigma \cdot \bar{\zeta}), \theta+\zeta, \bar{\theta}+\bar{\zeta}) \\
& =\frac{\partial}{\partial \bar{\theta}^{\dot{\alpha}}}\left[\left(\frac{\partial}{\partial \xi^{\alpha}}+i \sigma_{\alpha \dot{\beta}}^{v} \bar{\zeta}^{\dot{\beta}} \frac{\partial}{\partial X^{v}}\right) V(X, \xi, \bar{\xi})\right]_{\substack{X=x-i(\zeta \cdot \sigma \cdot \bar{\theta}-\theta \cdot \sigma \cdot \bar{\zeta}) \\
\xi=\theta+\zeta, \bar{\xi}=\bar{\theta}+\bar{\zeta}}} \\
& =\left[\left(\frac{\partial}{\partial \bar{\xi}^{\dot{\alpha}}}-i \zeta^{\beta} \sigma_{\beta \dot{\alpha}}^{\mu} \frac{\partial}{\partial X^{\mu}}\right)\right. \\
& \left.\times\left(\frac{\partial}{\partial \xi^{\alpha}}+i \sigma_{\alpha \dot{\beta}}^{v} \bar{\zeta}^{\dot{\beta}} \frac{\partial}{\partial X^{v}}\right) V(X, \xi, \bar{\xi})\right]_{\substack{X=x-i(\zeta \cdot \sigma \cdot \bar{\theta}-\theta \cdot \sigma \cdot \bar{\zeta}), \xi=\theta+\zeta, \bar{\xi}=\bar{\theta}+\bar{\zeta}}},
\end{aligned}
$$

and therefore

$$
A_{\mu}^{\prime}(x, \zeta, \bar{\zeta})=-\frac{1}{2} \bar{\sigma}_{\mu}^{\alpha \alpha} \bar{D}_{\dot{\alpha}}^{(\zeta)} D_{\alpha}^{(\zeta)} V(x, \zeta, \bar{\zeta}) .
$$

From this point of view, (21-29) and (32-34) tell us explicitly how supertranslation of the components of $V$ induces corresponding superfields.

\section{References}

[1] R. Clarkson, A. M. Ghezelbash and R. B. Mann, "New Reducible Membrane Solutions in D=11 Supergravity," Journal of High Energy Physics, 2004.

[2] A. M. Ghezelbash, "Supergravity Solutions without
Tri-Holomorphic U (1) Isometries,” Physical Review D, Vol. 78, 2008, p. 126002.

[3] de B. Wit, H. Nicolai and H. Samtleben "Gauged Supergravities, Tensor Hierarchies and M-Theory,” Journal of High Energy Physics, Vol. 2, 2008, p. 044.

[4] G. Compère, K. Copsey, de S. Buyl and R. B. Mann, "Solitons in Five-Dimensional Minimal Supergravity: Local Charge, Exotic Ergoregions and Violations of the BPS Bound,” Journal of High Energy Physics, Vol. 12, No. 47, 2009, p. 46.

[5] U. Gran, J. Gutowski and G. Papadopoulos, "M-Theory Backgrounds with 30 Killing Spinors are Maximally Supersymmetric,” ARXIV, 2010.

[6] R. Britto, E. Buchbinder, F. Cachazo and B. Feng, "One-Loop Amplitudes of Gluons in SQCD," Physical Review D, Vol. 72, 2005, p. 27.

[7] R. Dick, "Implications of a Dilaton in Gauge Theory and Cosmology," Fortschr der Physik, Vol. 45, 1997, pp. 537-587.

[8] R. Dick, "Heterotic String Dark Matter from the Graviton Multiplet," Physics Letters B, Vol. 510, 2001, pp. 10-16.

[9] J. McDonald and N. Sahu, "Kev Warm Dark Matter Via the Supersymmetric Higgs Portal,” Physical Review D, Vol. 79, 2009, p. 6.

[10] A. A. Bytsenko, S. D. Odintsov and S. Zerbini, "The Effective Action in Gauged Supergravity on Hyperbolic Background and Induced Cosmological Constant," Physics Letters B, Vol. 336, 1994, pp. 355-361.

[11] E. Elizalde, S. Jhingan, S. Nojiri, S. D.Odintsov, M. Sami and I. Thongkool, "Dark Energy Generated From a (Super) String Effective Action with Higher Order Curvature Corrections and a Dynamical Dilaton,” European Physical Journal C, Vol. 53, 2008, pp. 447-457.

[12] A. E. Nelson and N. Seiberg, "R-symmetry Breaking Versus Supersymmetry Breaking,” Nuclear Physics B, Vol. 416, 1994, 46-62.

[13] K. A. Intriligator, N. Seiberg and D. Shih, Supersymmetry Breaking, R-symmetry Breaking and Metastable Vacua,” Journal of High Energy Physics, Vol. 7, 2007, p. 19.

[14] M. Dine, G. Festuccia and Z. Komargodski, “A Bound on the Superpotential,” ARXIV, 2009, p. 2527.

[15] J. Wess and J. Bagger, "Supersymmetry and Supergravity, 2nd Edition,” Princeton University Press, 1992.

[16] J. Wess and B. Zumino, "Supergauge Transformations in four Dimensions,” Nuclear Physics B, Vol. 70, pp. 39-50; “A Lagrangian Model Invariant under Supergauge Transformations," Physics Letters B, Vol. 49, 1974, pp. 52-54.

[17] A. Salam and J. Strathdee, "Supergauge Transformations,” Nuclear Physics B, Vol. 76, 1974, pp. 477-482.

[18] S. Ferrara, J. Wess and B. Zumino, "Supergauge Multiplets and Superfields,” Physics Letters B, Vol. 51, 1974, pp. 239-241.

[19] A. Salam and J. Strathdee, "On Superfields and Fermi-Bose Symmetry,” Physical Review D, Vol. 11, 1975, pp. 1521-1535. 


\section{Appendix}

\section{A.1. Translation of Our Results Into the Conventions of Wessand Bagger}

Our superspace realizations (18) and (30) are related to the realizations in Wess and Bagger [15] according to

$$
\begin{aligned}
& Q_{\alpha}=-i Q_{\alpha}^{(W B)}, \bar{Q}_{\dot{\alpha}}=-i \bar{Q}_{\dot{\alpha}}^{(W B)}, \\
& D_{\alpha}=-i D_{\alpha}^{(W B)}, \bar{D}_{\dot{\alpha}}=-i \bar{D}_{\dot{\alpha}}^{(W B)} .
\end{aligned}
$$

With these conventions the component field expansions for chiral superfields agree and the generators for supertranslations are also the same (cf. $(4.11,4.12)$ in [15]),

$$
i(\zeta \cdot Q+\bar{Q} \cdot \bar{\zeta})=\zeta \cdot Q^{(W B)}+\bar{Q}^{(W B)} \cdot \bar{\zeta},
$$

i.e. our results for supertranslations to all orders also directly apply as generalizations of the first order transformation laws reported in [15].

Note that Wess and Bagger use an operator representation of the super-Poincare algebra with the same signature of the Minkowski metric but $p_{\mu}^{(W B)}=i \partial_{\mu}=-p_{\mu}$. This comes from the familiar sign difference between field theoretic and quantum mechanical operator realizations of Noether charges. If $\phi(x)=\langle x \mid \phi\rangle$ is a field operator, the momentum operators

$$
P_{\mu}=\int d^{3} x\left(\eta_{\mu}{ }^{0} \mathcal{L}-\partial_{\mu} \phi \cdot \frac{\partial \mathcal{L}}{\partial\left(\partial_{0} \phi\right)}\right)
$$

satisfy

$$
\left[P_{\mu}, \phi(x)\right]=i \partial_{\mu} \phi(x)
$$

and generate translations according to

$$
\begin{aligned}
& \phi^{\prime}(x)=\exp (-i \varepsilon \cdot P) \phi(x) \exp (i \varepsilon \cdot P)=\phi(x+\varepsilon) \\
& =\langle x+\varepsilon \mid \phi\rangle=\langle x|\exp (i \varepsilon \cdot p)| \phi\rangle=\left\langle x\left|\exp \left(-i \varepsilon \cdot p^{(\text {(WB })}\right)\right| \phi\right\rangle .
\end{aligned}
$$

Similar relations hold for Lorentz and gauge charges. Another way to look at the sign difference is through Jacobi identities. If the generators $X_{a}$ satisfy the Lie algebra,

$$
\left[X_{a}, X_{b}\right]=i C_{a b}{ }^{c} X_{c},
$$

then the adjoint matrix representation is given by

$$
\left(X_{a}\right)_{b}{ }^{c}=-i C_{a b}{ }^{c} \text {. }
$$

\section{A.2. Spinor Identities}

There are several useful identities for products of spinors which are used in the determination of the general linear multiplet and its transformation laws.

The following identities are a direct consequence of the anti-commutation properties of spinors and the definitions (9) and (10) of spinor products,

$$
\begin{gathered}
\theta^{\alpha} \theta^{\beta}=\varepsilon^{\alpha \beta} \theta^{1} \theta^{2}=\frac{1}{2} \varepsilon^{\alpha \beta}\left(-\theta^{1} \theta_{1}+\theta_{2} \theta^{2}\right) \\
=-\frac{1}{2} \varepsilon^{\alpha \beta} \theta^{\gamma} \theta_{\gamma}=-\frac{1}{2} \varepsilon^{\alpha \beta} \theta^{2}, \\
\bar{\theta}^{\dot{\alpha}} \bar{\theta}^{\dot{\beta}}=\varepsilon^{\dot{\alpha} \dot{\beta}} \bar{\theta}^{\mathrm{i}} \bar{\theta}^{\dot{2}}=\frac{1}{2} \varepsilon^{\dot{\alpha} \dot{\beta}} \bar{\theta}^{2}, \\
\theta_{\alpha} \theta^{\beta}=-\theta^{\beta} \theta_{\alpha}=-\frac{1}{2} \delta_{\alpha}^{\beta} \theta^{2}, \quad \bar{\theta}_{\dot{\alpha}} \bar{\theta}^{\dot{\beta}}=-\bar{\theta}^{\dot{\beta}} \bar{\theta}_{\dot{\alpha}}=\frac{1}{2} \delta_{\dot{\alpha}}^{\dot{\beta}} \bar{\theta}^{2} .
\end{gathered}
$$

Please note that $\theta^{2}$ in the first line in Equation (35) denotes the $\theta^{\alpha=2}$ component of the spinor $\theta$, but in the second line it is $\theta^{2}=\theta \cdot \theta$. In every equation in supersymmetry it is clear from the context what $\theta^{2}$ means. In all equations in previous sections of this paper $\theta^{2}$ always refers to $\theta^{2}=\theta \cdot \theta$.

The following relations also use the properties (3-7) of the Pauli matrices,

$$
\begin{gathered}
\psi \cdot \sigma^{\mu} \cdot \bar{\chi}=-\bar{\chi} \cdot \bar{\sigma}^{\mu} \cdot \psi, \\
\psi \cdot \sigma^{\mu} \cdot \bar{\sigma}^{v} \cdot \chi=\chi \cdot \sigma^{v} \cdot \bar{\sigma}^{\mu} \cdot \psi, \\
\bar{\theta}^{\dot{\alpha}} \psi^{\alpha}=\frac{1}{2} \bar{\sigma}_{\mu}^{\alpha \alpha} \psi \cdot \sigma^{\mu} \cdot \bar{\theta}, \\
\zeta \cdot \sigma^{\mu} \cdot \bar{\theta} \zeta \cdot \sigma^{\nu} \cdot \bar{\theta}=-\frac{1}{2} \eta^{\mu v} \zeta^{2} \bar{\theta}^{2} .
\end{gathered}
$$

